\title{
A Safe Surgical Procedure for Old Distractive Flexion Injuries of the Subaxial Cervical Spine
}

\author{
Osamu Kawano, Takeshi Maeda, Eiji Mori, Itaru Yugue, Takayoshi Ueta, Keiichiro Shiba \\ Department of Orthopaedic Surgery, Spinal Injuries Center, Fukuoka, Japan
}

\begin{abstract}
Study Design: Retrospective review.
Purpose: To describe a safe and effective surgical procedure for old distractive flexion (DF) injuries of the subaxial cervical spine. Overview of Literature: Surgical treatment is required in old cases when a progression of the kyphotic deformity and/or persistent neck pain and/or the appearance of new neurological symptoms are observed. Since surgical treatment is more complicated and dangerous in old cases than in acute distractive-flexion cases, the indications for surgery and the selection of the surgical procedure must be carefully conducted.

Methods: To identify a safe and effective surgical procedure, the procedure selected, reason(s) for its selection, and associated neurological complications were investigated in 13 patients with old cervical DF injuries.

Results: No neurological complications were observed in nine patients (DF stage 2 or 3) who underwent the anterior-posterior-anterior (A-P-A) method and two patients (DF stage 1) who underwent the posterior method. It was initially planned that two patients (DF stage 2) who underwent the P-A method would be treated using the Posterior method alone; however, anterior discectomy was added to the procedure after the development of a severe spinal cord disorder.

Conclusions: The A-P-A method (anterior discectomy, posterior release and/or partial facetectomy, reduction and instrumentation, anterior bone grafting) is considered to be a suitable surgical procedure for old cervical DF injuries.
\end{abstract}

Keywords: Old cervical spine injuries; Distractive flexion injuries; Post-traumatic deformity; Circumferential release; Delayed presentation

\section{Introduction}

Distractive flexion (DF) injuries [1] are often observed among patients with subaxial cervical spine injuries. The treatment generally selected for patients with such injuries is closed or open reduction and internal fixation with the purpose of spinal reconstruction, ensuring protection of the spinal cord. Reduction is usually performed during the acute phase, and good alignment can usually be obtained. However, the surgical treatment of patients with old injuries, where the injury was overlooked at the initial assessment, can be complicated and dangerous in terms of spinal alignment and spinal cord safety $[2,3]$. Surgical treatment is required in patients with old injuries when there is progression of the kyphotic deformity and/ or persistent neck pain and/or the appearance of new

Received Sep 21, 2016; Revised Nov 30, 2016; Accepted Dec 26, 2016

Corresponding author: Osamu Kawano

Department of Orthopaedic Surgery, Spinal Injuries Center, 550-4 Igisu, Iizuka, Fukuoka 820-8508, Japan

Tel: +81-948-24-7500, Fax: 81-948-29-1065, E-mail: orthosic@orange.ocn.ne.jp 
neurological symptoms [4]. Because surgical treatment is more complicated and dangerous in patients with old injuries than in those with acute DF injuries, indications for surgery and the selection of an appropriate surgical procedure must be carefully conducted.

The purpose of this study was to clarify which surgical procedure was the safest and most effective for treating patients with old DF injuries of the subaxial cervical spine. We also investigated the reasons why this type of injury is sometimes overlooked at the initial assessment and discuss the initial diagnostic methods that can be used to avoid overlooking such injuries in the future.

\section{Materials and Methods}

The medical records of all 1,208 patients with subaxial cervical spinal injuries who were surgically treated at our institute between 1990 and 2015 were retrospectively reviewed. Of these patients, only 13 were surgically treated and had old trauma injuries, which were all classified as DF injuries [1]. All patients were male and ranged in age from 23 years to 73 years. The mean duration of follow-up was 2.8 years (range, $2-5$ years).

To investigate the reasons why DF injuries were overlooked at the initial assessment, eventually resulting in old fracture dislocation or post-traumatic kyphotic deformities requiring treatment, the following four items were obtained from the records of the 13 patients: (1) the affected intervertebral segment, (2) the presence of neck pain and neurological symptoms at the initial assessment, (3) the use of radiography at the initial assessment and the detection of any abnormalities on available radiographs, and (4) the time between injury and surgery and the symptoms that led to the correct diagnosis. To investigate the suitability of the surgical procedure, the following items were investigated: (5) the surgical procedure and the reason(s) for its selection and (6) neurological and any other complications associated with surgery.

\section{Results}

\section{Affected segment of the cervical spine}

Among the 13 patients, the $C 4 / 5$ segment was affected in three, the C5/6 segment in four, and the C6/7 segment in six (Table 1). Many of the injuries affected the lower cervical spine, where injuries are prone to being overlooked during radiography because of overlap with the shoulder. One patient with a C4/5 injury was diagnosed with a DF injury at the initial assessment at another hospital; however, the patient was conservatively treated using a halo vest to obtain reduction and fibrous fusion. Another patient with a C4/5 injury had a complication of a dislocated larynx; treatment for this delayed the treatment for cervical spine dislocation. A third patient with a C4/5 injury had subluxation that spontaneously repositioned in the supine

Table 1. Case summary: primary assessment

\begin{tabular}{|c|c|c|c|c|c|}
\hline Case & Level & Neck pain & Neurological symptom & Radiographic examination & Radiographic abnormality \\
\hline 1 & C6/7 & + & Arm pain & + & None \\
\hline 2 & $\mathrm{C} 4 / 5$ & - & - & + & + (Halo vest) \\
\hline 3 & $\mathrm{C} 5 / 6$ & + & - & + & None \\
\hline 4 & C5/6 & + & - & - & - \\
\hline 5 & C6/7 & & - & + & None \\
\hline 6 & C5/6 & + & - & - & - \\
\hline 7 & C6/7 & - & - & - & - \\
\hline 8 & C6/7 & + & - & + & None \\
\hline 9 & $\mathrm{C} 5 / 6$ & + & Arm pain & + & None \\
\hline 10 & $\mathrm{C} 6 / 7$ & + & Quadriplegia & + & None \\
\hline 11 & $\mathrm{C} 4 / 5$ & + & - & + & + (Dislocation of the larynx) \\
\hline 12 & $\mathrm{C} 4 / 5$ & + & - & + & None \\
\hline 13 & C6/7 & + & - & + & CT: C7 fracture \\
\hline
\end{tabular}

$\mathrm{CT}$, computed tomography. 
position at the initial assessment.

\section{Symptoms immediately following injury}

Neck pain was observed at the initial assessment in $10 \mathrm{pa}-$ tients, with some type of neurological symptoms observed in three patients (two with arm pain and one with incomplete quadriplegia) (Table 1).

\section{Radiography at the initial assessment}

Radiography was not performed at the initial assessment in three patients (Table 1). Of the 10 patients examined by radiography, abnormal findings were observed in only two: the patient treated with a halo vest and the patient with the laryngeal injury, described above. A patient was diagnosed as having a C7 vertebral body fracture by a computed tomography (CT) scan. No abnormalities were observed in seven patients who were radiographically ex-

Table 2. Case summary: primary and new symptoms

\begin{tabular}{lcc} 
Case & Primary symptoms (persistence or aggravation) & New symptoms \\
\hline 1 & Neck pain & - \\
\hline 2 & Neck pain (halo vest) & ROM limitation of the neck \\
3 & Neck pain & Numbness of the arm \\
\hline 4 & Neck pain & Neck and arm pain \\
\hline 5 & - & Numbness of the fingers \\
\hline 6 & - & Neck pain and palsy of U/E \\
\hline 7 & - & Palsy of U/E \\
\hline 8 & Neck pain & - \\
\hline 9 & Neck pain and arm pain & Ouadriplegia \\
\hline 10 & Neck pain (dislocation of larynx case) & - \\
\hline 12 & Neck pain & Lt. C5 palsy \\
\hline 13 & Neck pain & Lt. arm pain
\end{tabular}

ROM, range of motion; U/E, upper extremity; Lt., left.

Table 3. Case summary: diagnosis, treatment, and complications

Case Time between injury and surgery (mo) Reduction prior to surgery Surgical method Neurological deterioration

\begin{tabular}{llccc}
1 & 1 & + & $P$ & - \\
2 & 5 & - & A-P-A & - \\
3 & 4.5 & - & A-P-A & - \\
4 & 1.5 & + & $P$ & - \\
5 & 1.5 & - & A-P-A & - \\
6 & 3 & - & A-P-A & - \\
7 & 3 & - & A-P-A & AIS E $\rightarrow$ A $\rightarrow$ D \\
\hline 8 & 2 & - & P-A & - \\
\hline 9 & 2 & - & A-P-A & AIS D D A A C \\
10 & 3.5 & - & A-P-A & - \\
11 & 1.5 & - & A-P-A & - \\
\hline
\end{tabular}

P, posterior; A anterior; P-A, posterior-anterior; AIS, American Spinal Injury Association Impairment Scale. 
amined. It was thought that these cases included patients in whom the subluxation became spontaneously repositioned or the affected segment was hidden by the shoulder and could not be diagnosed.

\section{Time between injury and surgery and key symptoms that led to the correct diagnosis}

Table 2 lists the patients' primary and new symptoms, and Table 3 summarizes their diagnoses and treatment. The time interval between injury and surgery was one to five months (mean, 2.4 months). Nine patients were diagnosed on the basis of other radiological examinations investigating new symptoms, and two patients were diagnosed upon reinvestigation because of symptoms that had persisted since the initial assessment.

\section{Surgical procedures}

The anterior-posterior-anterior (A-P-A) method (anterior discectomy, posterior release and/or partial facetectomy, reduction and instrumentation, and anterior bone grafting) (Fig. 1) was performed for nine patients (five at DF stage 2 and four at DF stage 3); the posterior-anterior (PA) method (posterior release and/or partial facetectomy reduction and instrumentation, anterior discectomy, and bone grafting) was performed for two patients, both at DF stage 2; and the posterior (P) method (posterior release, reduction, and fusion) was performed for two patients, both at DF stage 1 (Table 3 ). Either spinous process wiring or the lateral mass screw and rod system was used for posterior instrumentation. Lateral flexion-extension radiography using $\mathrm{X}$-ray images obtained prior to surgery
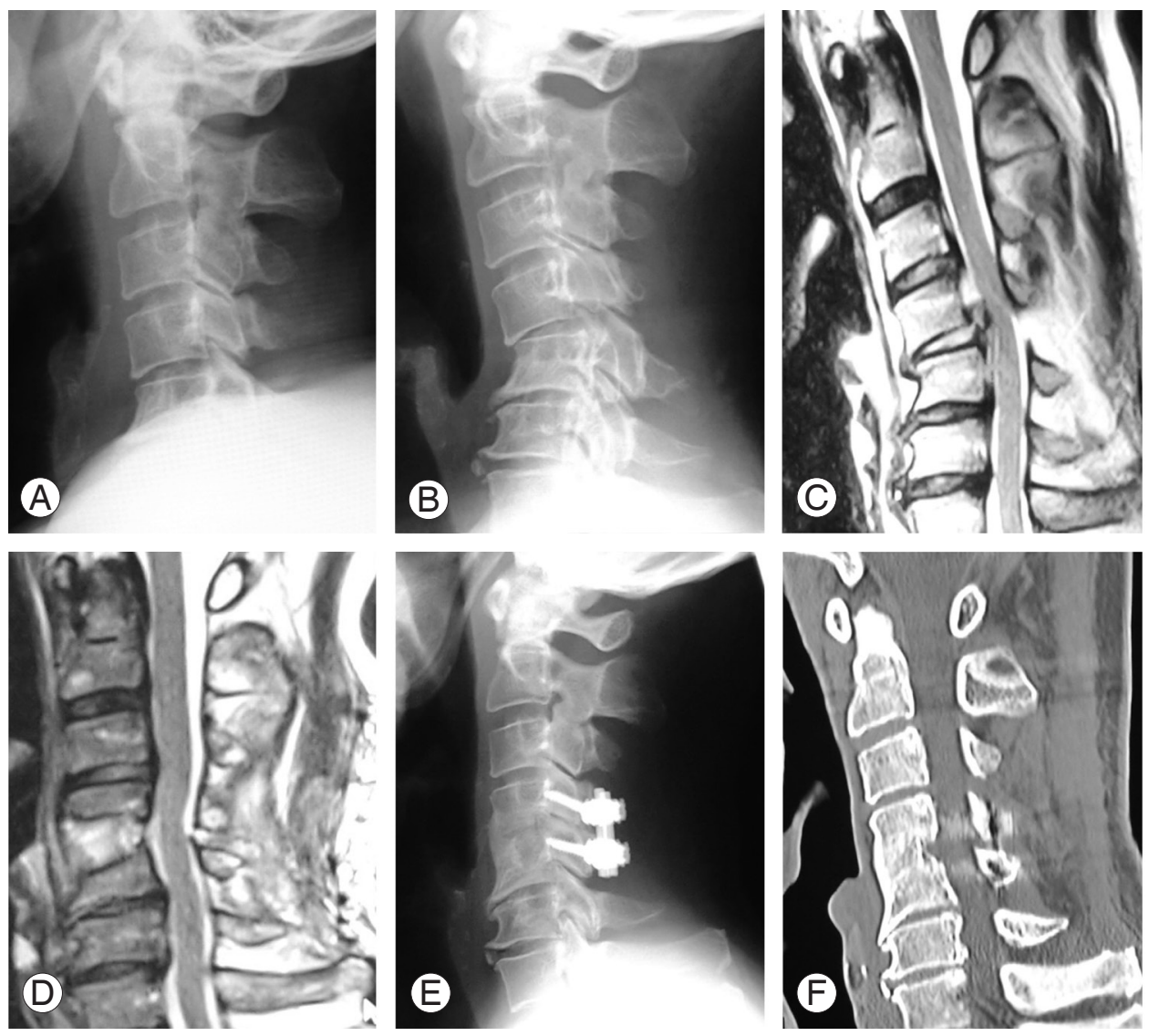

Fig. 1. (A) Lateral X-ray obtained immediately after the injury (supine position). The distractive flexion (DF) injury was not clear. (B) Lateral X-ray obtained after three weeks (sitting position). C4/5 subluxation due to the DF injury has been cleared. The patient's neck pain persisted and left C5 palsy subsequently developed. (C) Magnetic resonance imaging (MRI): T2 sagittal image. The C4/5 disc migrated to the spinal canal. (D) MRI: T2 sagittal image. Disc herniation was removed with sufficient spinal cord decompression. (E) Lateral X-ray obtained after surgical treatment with the anterior-posterior-anterior method using the lateral mass screw system and anterior iliac bone grafting. Good alignment was obtained. (F) Multi planar reconstruction-computed tomography (one year after surgery). Good bony fusion was obtained at the $\mathrm{C} 4 / 5$ interve rtebral body. 

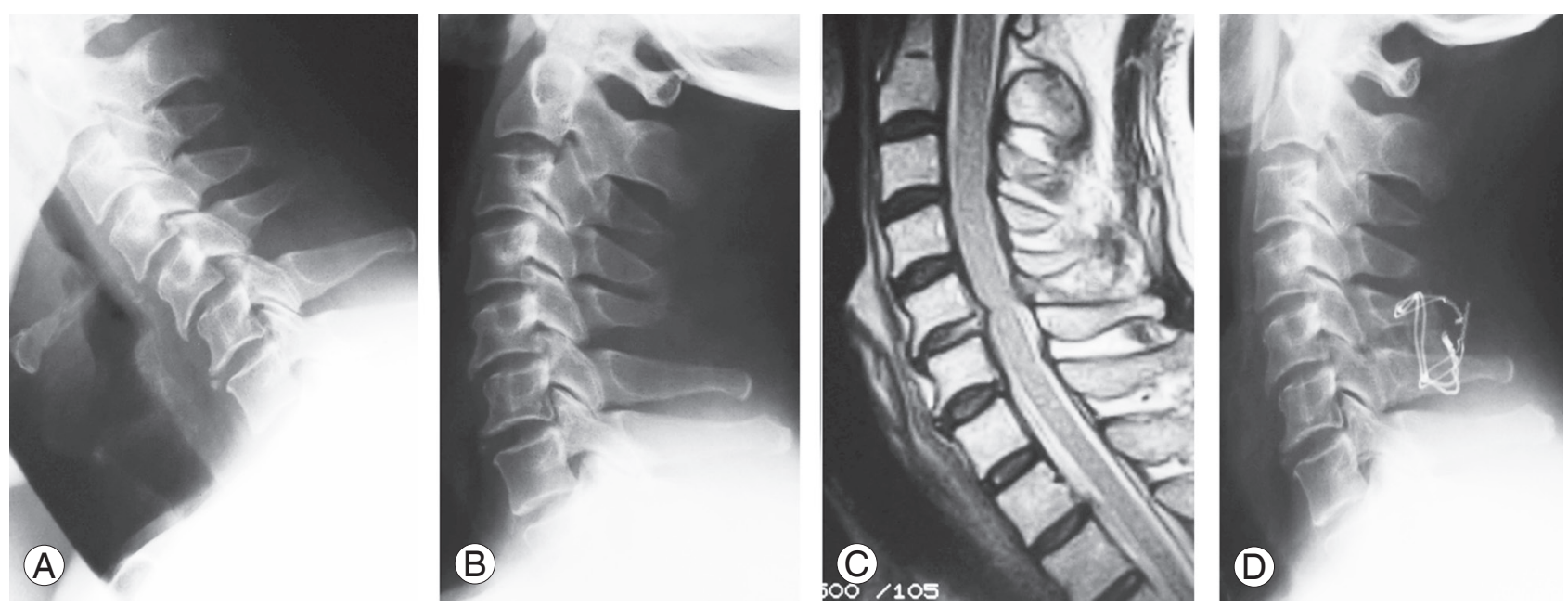

Fig. 2. (A) Lateral flexion X-ray: C5/6 distractive flexion injury. C5 subluxation (DFS1). (B) Lateral extension X-ray: a reduction position was obtained with no neurological deterioration. (C) Magnetic resonance imaging (MRI): T2 sagittal image. No neurological complications occurred despite C5/6 disc bulging. (D) Lateral X-ray obtained after surgical treatment: posterior reduction and fixation with spinous process wiring and bone grafting were performed.

confirmed that both patients treated with the P method alone had obtained a reduction. Radiography had been performed with the assistance of a spine surgeon while the patient was conscious, and the lack of any new neurological symptoms (spinal cord disorder or radiculopathy) was confirmed at that time (Fig. 2).

It was initially planned that the two patients who underwent the P-A method would be treated using the P method alone; however, these patients developed severe spinal cord disorders. Therefore, anterior decompression with discectomy and bone grafting were also performed. Neither patient achieved reduction, as indicated by lateral flexion-extension radiography using $\mathrm{X}$-ray images prior to surgery.

\section{Outcomes and complications}

There were no intraoperative or postoperative complications among the nine patients treated using the A-P-A method or the two patients treated using the P method. These patients obtained good bony fusion. As described above, the anterior method was added to the treatment regimen of two patients because of the development of severe spinal cord disorders. In both patients, aggravation of the spinal cord disorder was caused by increased spinal cord compression due to intervertebral disc herniation. In one of these patients, the neurological status deteriorated from American Spinal Injury Association Impairment Scale (AIS) E to AIS A immediately after posterior surgery. However, one year after undergoing immediate anterior decompression surgery, the patient's neurological status had recovered to AIS D (Fig. 3). The other patient's neurological status was AIS D before surgery. Because neurological deterioration from AIS D to AIS A was noted immediately after posterior surgery, emergency anterior decompression surgery was subsequently performed. At the final follow-up visit two years after presentation, the patient's neurological status was found to have recovered to only AIS C.

The mean surgical time was 229.3 minutes (range, 160325 minutes) for the A-P-A method, 64.0 minutes (range, 60-68 minutes) for the P method, and 201.5 minutes (range, 178-225 minutes) for the P-A method. The mean blood loss was $269.2 \mathrm{~g}$ (range, $89-538 \mathrm{~g}$ ) for the A-P-A method, $99.5 \mathrm{~g}$ (range, 80-119 g) for the P method, and $152.5 \mathrm{~g}$ (range, 108-197 g) for the P-A method.

\section{Compliance with ethical standards}

This article does not contain any studies with human participants or animals performed by any of the authors. Informed consent was obtained from all individual participants included in the study.

\section{Discussion}

Two problems can arise regarding old cervical DF injuries. The first relates to the initial assessment, and the second 

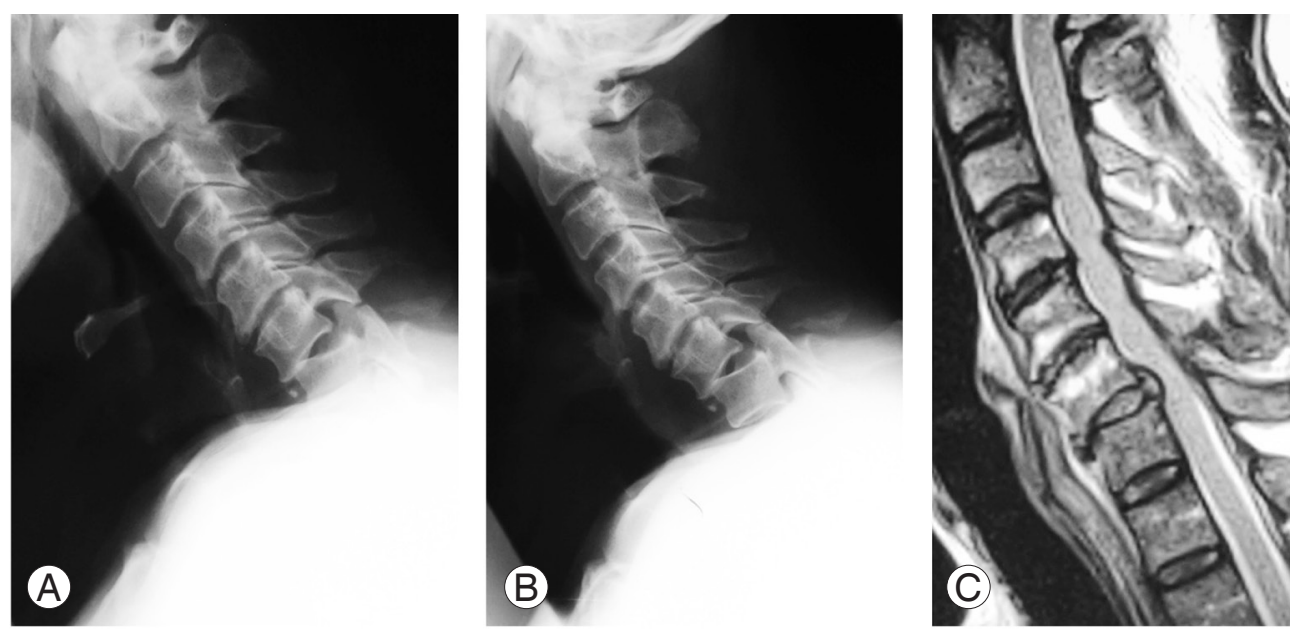

Fig. 3. (A) Lateral neutral X-ray: C6/7 distractive flexion injury. (B) Lateral extension $\mathrm{X}$-ray: a reduction position could not be obtained. (C) Magnetic resonance imaging (MRI): T2 sagittal image. C6/7 disc protrusion. (D) Lateral $X$-ray after surgical treatment: anterior decompression and bone grafting were added to the treatment regimen immediately following posterior surgery with release, reduction, and fixation because of the development of neurological deterioration as a result of disc herniation after surgery. (E) MRI: T2 sagittal image obtained after surgery. A high-intensity area remained in the spinal cord.
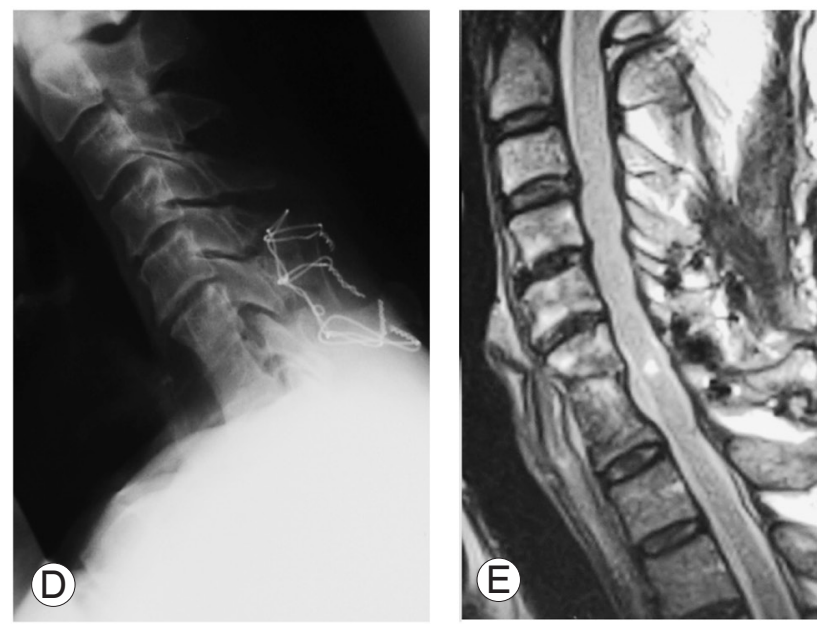

relates to the surgical procedure. The initial diagnosis and treatment are performed as emergency medical services, where it is difficult to sufficiently evaluate the lower cervical spine because it is hidden by the shoulder $[5,6]$. CT scanning has become simplified in recent years, allowing the evaluation of bone fractures in the lower cervical spine. However, spontaneous reduction of anterior dislocation or subluxation without fractures in the lower cervical spine can sometimes occur in association with DF injuries, but these cannot be accurately evaluated using CT [7]. When a cervical spine injury is suspected based on the circumstances under which the injury occurred or because of the presence of symptoms such as severe neck pain, it is necessary to carefully apply lateral flexionextension radiography using X-ray images, with the assistance of a spine surgeon, in addition to radiography and CT scans.

In patients in whom the affected segment of the cervical spine is hidden by the shoulder, it is worth evaluating using swimmer's lateral view flexion-extension radiography or a CT scan in the flexion position. At our institute, lateral flexion-extension radiography or CT scans in the flexion position are performed during the initial assessment and the degree of instability due to soft tissue injury is evaluated [8]. These investigations should be carefully performed such that the patient's neurological symptoms are not aggravated. Recently, many surgeons have been using magnetic resonance imaging (MRI) to detect soft tissue injuries. The frequency of performing stress radiography is therefore expected to decrease in the future.

Our patients were surgically treated because of the persistence of neck and arm pain or the deterioration of neurological symptoms [4]. These symptoms were caused by progression of the kyphotic deformity and/or persistent instability of the cervical spine. Accordingly, when selecting the surgical procedure, the goal should be to achieve both good alignment and bony fusion to reconstruct the injured cervical spine and avoid worsening any neurologi- 
cal symptoms.

Previous reports have discussed surgical procedures for old (or delayed presentation) DF injuries, with apparent advantages and disadvantages for each of these methods [2,9-11]. Bartels and Donk [2] concluded that the A-P-A method should be performed for patients with non-acute bilateral cervical facet dislocations. Hassan [10] reported a method involving five steps, with traction in between; however, the method is very complicated and leads to a long treatment course. Liu et al. [11] reported that anatomical reduction can be successfully achieved with the P-A method, primarily including posterior release, anterior release, reduction, intervertebral bone grafting, and anterior plating. However, we are concerned about the possibility of the failure of reduction via the anterior method and the low stability provided by anterior plating for circumferential soft tissue release to the cervical spine. Basu et al. [12] reported that preoperative traction is a safe and effective initial treatment for patients with neglected cervical facet dislocation. Traction may be effective for acute and subacute patients; however, the patients in that study were treated at 7 to 21 days (mean, 14 days) after injury, whereas our patients were treated more than one month after the injury. Goni et al. [13] suggested that there was no role for skull traction in neglected DF injuries to the cervical spine after a delay of more than three weeks. They recommended the Posterior method followed by the anterior method.

Based on our experience, surgery can be safely performed without worsening the patient's neurological symptoms using the A-P-A method (anterior discectomy, posterior release and/or partial facetectomy, reduction and instrumentation, and anterior bone grafting), and we believe that this method should therefore be recommended, even though it is rather complex to perform. Based on their experience, Liu et al. [11] recommended either the $\mathrm{P}-\mathrm{A}$ method (posterior release, anterior release, reduction, intervertebral grafting, and anterior plating) or the P-A-P method (posterior release, anterior reduction and plate fixation, and posterior instrumentation). However, the Posterior method has advantages in terms of safety and strength for instrumentation during the reduction and for arranging spinal alignment. We therefore consider the A$\mathrm{P}$-A method to be more reasonable and to able to provide better outcomes than the P-A and P-A-P methods. In any case, circumferential release, fixation, and bone grafting are likely to be necessary in patients with old DF injuries

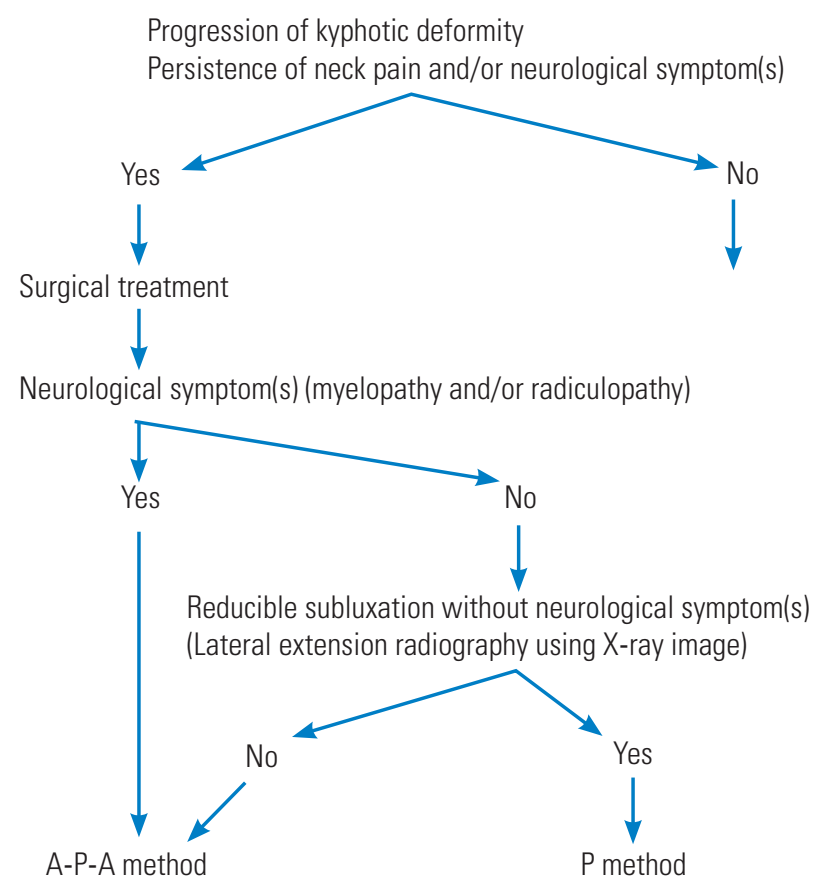

Fig. 4. Flowchart for selecting the surgical method for treating patients with old distractive flexion injuries. A-P-A, anterior-posterioranterior; P, posterior.

associated with neurological symptoms and/or kyphotic deformities.

The $\mathrm{P}$ method can be applied in patients at DF stage 1; using this method, good reduction is achieved on lateral extension radiography using X-ray images prior to surgery, without worsening the patient's neurological symptoms or disc herniation on MRI (Fig. 4).

Our experience of patients with old cervical DF injuries in which one or more months have passed since the trauma has shown that forcible reduction via posterior release alone results in an excess load to the anterior element (intervertebral disc) during the process of scar formation, bringing the risk of deterioration of the patient's neurological symptoms due to herniation of the intervertebral discs [14]. The optimal type of surgical management should be determined not only by the label of "old" but also based on whether there is an irreducible condition due to scar formation resulting from the microinstability of an old DF injury. The A-P-A method is considered to be a reasonable, safe, and reliable procedure that can be applied for patients with old DF injuries of the subaxial cervical spine. The $\mathrm{P}$ method can be applied, such as in patients at DF stage 1 , in whom good reduction is achieved on lateral extension radiography using $\mathrm{X}$-ray images prior to sur- 
gery, without a deterioration in the patient's neurological symptoms or disc herniation on MRI.

A limitation of the present study was the small population size (only 13 patients). However, this was inevitable, as this kind of lesion is infrequent. We nevertheless hope that our clinical experience can help spine surgeons safely treat patients with old DF injuries.

\section{Conclusions}

Because surgical treatment is more complicated and dangerous for patients with old DF injuries than for those with acute DF injuries, the surgical method must be carefully considered. Circumferential release, fixation, and bone grafting of the spinal column are likely to be necessary in patients with old cervical DF injuries associated with neurological symptoms and/or kyphotic deformities. The A-P-A method (anterior discectomy, posterior release and/or partial facetectomy, reduction and instrumentation, and anterior bone grafting) is considered to be a reasonable, safe and effective surgical procedure for treating patients with old cervical DF injuries.

\section{Conflict of Interest}

No potential conflict of interest relevant to this article was reported.

\section{References}

1. Allen BL Jr, Ferguson RL, Lehmann TR, O’Brien RP. A mechanistic classification of closed, indirect fractures and dislocations of the lower cervical spine. Spine (Phila Pa 1976) 1982;7:1-27.

2. Bartels RH, Donk R. Delayed management of traumatic bilateral cervical facet dislocation: surgical strategy: report of three cases. J Neurosurg 2002;97:362-5.

3. Kahn A, Leggon R, Lindsey RW. Cervical facet dislocation: management following delayed diagnosis.
Orthopedics 1998;21:1089-91.

4. Vaccaro AR, Silber JS. Post-traumatic spinal deformity. Spine (Phila Pa 1976) 2001;26:S111-8.

5. Bohlman HH. Acute fractures and dislocations of the cervical spine: an analysis of three hundred hospitalized patients and review of the literature. J Bone Joint Surg Am 1979;61:1119-42.

6. Kwon BK, Vaccaro AR, Grauer JN, Fisher CG, Dvorak MF. Subaxial cervical spine trauma. J Am Acad Orthop Surg 2006;14:78-89.

7. Kolli S, Schreiber A, Harrop J, Jallo J. Traumatic cervical spinal cord injury with "negative" cervical spine CT scan. BMJ Case Rep 2010;2010:pii:bcr12. 2009.2525.

8. Maeda T, Ueta T, Mori E, et al. Soft-tissue damage and segmental instability in adult patients with cervical spinal cord injury without major bone injury. Spine (Phila Pa 1976) 2012;37:E1560-6.

9. Allred CD, Sledge JB. Irreducible dislocations of the cervical spine with a prolapsed disc: preliminary results from a treatment technique. Spine (Phila Pa 1976) 2001;26:1927-30.

10. Hassan MG. Treatment of old dislocations of the lower cervical spine. Int Orthop 2002;26:263-7.

11. Liu P, Zhao J, Liu F, Liu M, Fan W. A novel operative approach for the treatment of old distractive flexion injuries of subaxial cervical spine. Spine (Phila $\mathrm{Pa}$ 1976) 2008;33:1459-64.

12. Basu S, Malik FH, Ghosh JD, Tikoo A. Delayed presentation of cervical facet dislocations. J Orthop Surg (Hong Kong) 2011;19:331-5.

13. Goni V, Gopinathan NR, Krishnan V, Kumar R, Kumar A. Management of neglected cervical spine dislocation: a study of six cases. Chin J Traumatol 2013;16:212-5.

14. Sim E, Vaccaro AR, Berzlanovich A, Schwarz N, Sim B. In vitro genesis of subaxial cervical unilateral facet dislocations through sequential soft tissue ablation. Spine (Phila Pa 1976) 2001;26:1317-23. 